\title{
Utilização de frutos de cactos (Cactaceae) como recurso alimentar por vespas sociais (Hymenoptera, Vespidae, Polistinae) em uma área de caatinga (Ipirá, Bahia, Brasil)
}

\author{
Gilberto M. M. Santos '; Jucelho D. da Cruz '; Carlos C. Bichara Filho '; \\ Oton M. Marques ${ }^{2} \&$ Cândida M. L. Aguiar ${ }^{1}$
}

\begin{abstract}
${ }^{1}$ Laboratório de Entomologia, Departamento de Ciências Biológicas, Universidade Estadual de Feira de Santana. 44031-460 Feira de Santana, Bahia, Brasil. E-mail: gmms@uefs.br

${ }^{2}$ Centro de Ciências Agrárias, Ambientais e Biológicas, Universidade Federal do Recôncavo da Bahia. 44380-000 Cruz das Almas, Bahia, Brasil.
\end{abstract}

\begin{abstract}
The use of cactus fruit food resources by social wasp (Hymenoptera, Vespidae, Polistinae) in an area of Caatinga (Ipirá, Bahia, Brazil). This study aims to identify food resource plants used by social wasp species in a Caatinga area, as well as describe the local patterns of cactus fruit resources used by wasps. Nine hundreds and eight foraging wasps, belonging to nine species, were captured while visiting six cactus species. Cereus jamacaru DC. and Pilosocereus catingicola (Gurke) Byles \& G.D. were the most frequently visited plants. Several wasp species and a great number of individuals visited them. Polybia paulista von Ihering, 1896, Polybia ignobilis (Haliday, 1836), Polistes versicolor (Olivier, 1791), Polistes simillimus Zikán, 1951, Polistes billardieri Fabricius, 1804, Polistes canadensis (Linnaeus, 1758), Polybia occidentalis (Olivier, 1791) and Polybia sericea (Olivier, 1705) had the larger trophic niche breadth. The highest trophic niche overlap was between Mischocyttarus lanei Zikán, 1949 and Polistes simillimus Zikán, 1951 (0.83). The high niche overlap among social wasps seems to be resulting of the generalist behavior of the social wasp, as well as of the intensive exploration of few cactus species.
\end{abstract}

KEY WORDS. Semi-arid; trophic niche; wasp assemblage.

RESUMO. Este estudo teve por objetivo identificar as espécies utilizadas como recurso alimentar e descrever os padrões locais de utilização de frutos de cactos como recurso alimentar por vespas sociais em uma área de Caatinga. Novecentos e oito indivíduos de nove espécies de vespas foram capturados visitando seis espécies de cactáceas. Cereus jamacaru DC. e Pilosocereus catingicola (Gurke) Byles \& G.D foram as cactáceas mais utilizadas por vespas sociais, tanto em número de espécies quanto de indivíduos. Polybia paulista von Ihering, 1896, Polybia ignobilis (Haliday, 1836), Polistes versicolor (Olivier, 1791), Polistes simillimus Zikán, 1951, Polistes billardieri Fabricius, 1804, Polistes canadensis (Linnaeus, 1758), Polybia occidentalis (Olivier, 1791) e Polybia sericea (Olivier, 1705) apresentaram as maiores amplitudes de nicho trófico. As maiores sobreposições no nicho trófico foram observadas entre Mischocyttarus lanei Zikán, 1949 e Polistes simillimus Zikán, $1951(0,83)$. Os altos níveis de sobreposição de nichos observados são explicados em função do comportamento generalista das vespas sociais, bem como da exploração intensiva de poucas espécies de cactáceas.

PALAVRAS-CHAVE. Comunidade de vespas; nicho trófico; Semi-árido.

A composição florística e a estrutura da vegetação são determinantes na composição e estrutura das comunidades de vespas sociais, influenciando diretamente em seus nichos fundamentais e realizáveis. A vegetação provê substrato de nidificação (SANTOS \& GobBi 1998, Cruz et al. 2006), recursos glucídicos (Santos et al. 1998, Pereira \& Santos 2006, Santos et al. 2006), material para construção de ninhos (MACHADO 1982, Marques \& Carvalho 1993) e área de caça (Santos et al. 1998). Algumas espécies de vespas só nidificam sob certas condições estruturais da vegetação, selecionando fisionomias abertas ou fechadas, bem como condições morfológicas das espécies vegetais, forma e tamanho de folhas, diâmetro do tronco e ou presença de espinhos (HenRiQues et al. 1992, SANTos \& GobBi 1998, Cruz et al. 2006).

Altamente sazonais, as Caatingas constituem a porção norte da "Diagonal Seca" e representam uma das mais importantes formações vegetais da América do Sul (Prado \& GibBS 1993). São caracterizadas pelo clima semi-árido e pela existên-

Revista Brasileira de Zoologia 24 (4): 1052-1056, dezembro 2007 
cia de longos períodos de baixa pluviosidade, que refletem em períodos de marcada escassez de recursos alimentícios para a fauna. Análises de disponibilidade de recursos e capacidade de suporte ambiental são particularmente importantes quando esses recursos mostram flutuações sazonais em sua disponibilidade (BEGON et al. 1996, SANTos et al. 2006).

Além de importante na manutenção de vários organismos vertebrados e invertebrados das regiões de Caatinga, as cactáceas apresentam importância econômica, podendo ser utilizadas na alimentação animal, na alimentação humana, como fonte de madeira e para o paisagismo (Lima 1996).

O objetivo deste estudo foi analisar a estrutura da guilda de vespas sociais que utilizam frutos de cactos, bem como identificar as espécies de cactos utilizadas como recurso alimentar por vespas sociais, em uma área de Caatinga do estado da Bahia.

\section{MATERIAL E MÉTODOS}

O estudo foi realizado na Fazenda Encantado, município de Ipirá ( $12^{\circ} 13^{\prime} \mathrm{S}$ e $\left.39^{\circ} 50^{\prime} \mathrm{W}\right)$, Bahia, Brasil. A localidade apresenta clima do tipo semi-árido, com temperatura média anual de $23.7^{\circ} \mathrm{C}$, pluviosidade média anual de $754 \mathrm{~mm}$ e mínima de 157 mm. (CEı 1994). A vegetação, submetida ao corte seletivo para implantação de pasto para criação de gado bovino, apresenta grande quantidade de cactáceas, árvores esparsas, predominância de arbustos e herbáceas.

Durante nove meses, entre setembro de 1999 e maio de 2000, período de pico da frutificação das cactáceas na região, foram realizadas observações mensais de vespas coletando recursos alimentares em frutos de cactos. Em cada amostragem, dois coletores percorriam continuamente uma área de 60 ha (7 às $18 \mathrm{~h}$ ) inspecionando e coletando vespas em frutos de Cactaceae. Adicionalmente, foram coletados dados sobre o número de colônias de vespas sociais de cada espécie e os tipos de substratos de nidificação utilizados na área amostrada.

A amplitude do nicho trófico das vespas foi calculada através do índice de SHANNON (1948), usando a fórmula $H^{\prime}=-\Sigma p_{k} \ln$ $\mathrm{p}_{\mathrm{k}^{\prime}}$ onde: $\mathrm{p}_{\mathrm{k}}$ é a proporção de indivíduos coletados na planta $\mathrm{K}$ e ln é o logaritmo neperiano do valor $\mathrm{p}_{\mathrm{k}}$. A equitabilidade das visitas de cada espécie de vespa aos frutos das espécies de cactos foi calculada através da fórmula $\mathrm{J}^{\prime}=\mathrm{H}^{\prime} / \mathrm{H}^{\prime}{ }_{\max }$ onde: $\mathrm{H}_{\max }^{\prime}$ é o logaritmo neperiano do número total de espécies de plantas visitadas pela espécie de vespa social.

A sobreposição dos nichos tróficos das espécies foi calculada pelo índice de Schoener (1982), usando a fórmula $\mathrm{NO}_{\text {ih }}=1-1 / 2 \Sigma_{\mathrm{k}}\left|\mathrm{p}_{\mathrm{ik}}-\mathrm{p}_{\mathrm{hk}}\right|$, onde: "i" e " $\mathrm{h}$ " representam as espécies de vespas comparadas, $\mathrm{p}_{\mathrm{ik}}$ e $\mathrm{p}_{\mathrm{hk}}$, são as proporções de indivíduos respectivamente das espécies de vespas " $\mathrm{i}$ " e " $\mathrm{h}$ " coletados na espécie de planta $\mathrm{k} . \mathrm{p}_{\mathrm{ik}}$ é obtida dividindo-se o número de indivíduos da espécie "i" coletado na planta k pelo número total de indivíduos de "i" obtidos em todas as plantas. Este índice permite analisar a sobreposição entre cada conjunto de duas espécies. Mischocyttarus lanei Zikán, 1949, foi excluído da análise, por ter sido representada por uma amostra muito pequena $(n=2)$.

\section{RESULTADOS E DISCUSSÃO}

Foram coletados 908 indivíduos, pertencentes a nove espécies de vespas sociais, coletando recursos alimentares em seis espécies de cactos (Tab. I). Cinco espécies de vespas, Polybia paulista von Ihering, 1896, Polybia sericea (Olivier, 1705), Polybia ignobilis (Haliday, 1836), Polistes canadensis (Linnaeus, 1758) e Polistes versicolor (Olivier, 1791) foram predominantemente encontradas na guilda de vespas sociais que utilizam frutos de cactos como recurso alimentar (808 indivíduos, 89,08\% do total). As espécies M. lanei, Polistes simillimus Zikán, 1951 e Polistes billardieri Fabricius, 1804 foram consideradas acidentais na comunidade de vespas sociais, apresentando freqüências inferiores a $1 \%$ do total de indivíduos coletados.

As vespas utilizaram os frutos de Cactaceae de forma diferenciada. O forrageamento de vespas nos frutos de cactos em Ipirá foi concentrado principalmente nas espécies Cereus jamacaru DC. e Pilosocereus catingicola, (Gurke) Byles \& G.D., as quais reuniram $71,58 \%$ do total de indivíduos amostrados, além de serem as únicas visitadas por todas as nove espécies de vespas sociais.

Por outro lado, os frutos das espécies Melocactus bahiensis (Britton \& Rose) Werderm e Opuntia palmadora Britton \& Rose foram muito pouco utilizados como recursos alimentares pelas vespas sociais $(1,22 \%$ dos indivíduos amostrados), indicando que, em termos comparativos, não apresentam importância na dieta das vespas sociais da Caatinga de Ipirá. As espécies Harrisia adscendens (Gurke) Britton \& Rose e Pilosocereus gounellei (Weber) Byles \& Rowley tiveram visitação intermediária sendo visitadas, em conjunto, por $27,20 \%$ dos indivíduos amostrados.

Dentre as espécies de vespas mais abundantes, apenas $P$. paulista foi coletada com abundância equivalente nas diferentes Cactaceae utilizadas. As demais espécies predominantes apresentaram variações, com dominância em uma das espécies vegetais amostradas (Tab. I). Polistes canadensis foi mais freqüente nos frutos de C. jamacaru $(\mathrm{F}=46 \%)$, $P$. sericea nos frutos de $P$. catingicola $(\mathrm{F}=57 \%), P$. versicolor nos de $H$. adscendens $(\mathrm{F}=46 \%)$ e $P$. ignobilis a mais freqüente nos frutos de P. gounelli $(\mathrm{F}=34 \%)$.

A sobreposição dos nichos tróficos entre as espécies de vespas sociais variou de 0 a 0,50 (Tab. II). As duas espécies mais abundantes na comunidade ( $P$. sericea e $P$. canadensis) apresentaram sobreposição baixa (15\%). A maioria (25 de 28) dos pares de espécies comparados apresentou sobreposição inferior a $30 \%$, que é considerada baixa, especialmente para vespas sociais. Apenas três dos 28 pares de espécies comparados apresentaram sobreposição correspondente a 50\%. Estes resultados diferem dos encontrados por Hermes \& KoHLer (2006), que observaram que espécies de vespas sociais que compartilham recursos florais, em geral, apresentam tendência à alta sobreposição do nicho trófico.

Dentre as espécies predominantes na guilda de vespas que utilizam frutos de cactos, as espécies $P$. ignobilis e $P$. paulista apresentaram maior amplitude de nicho trófico $\left(H^{\prime}=1,38\right)$. Essas espécies diferiram bastante das demais na estratégia de 
Tabela I. Vespas sociais coletadas em frutos de cactos em Ipirá, Bahia, de setembro de 1999 a maio 2000.

\begin{tabular}{|c|c|c|c|c|c|c|c|}
\hline \multirow[b]{2}{*}{ Vespas } & \multicolumn{6}{|c|}{ Espécies de cactáceas utilizadas } & \multirow[b]{2}{*}{ Total } \\
\hline & $\begin{array}{c}\text { Cereus } \\
\text { jamacaru }\end{array}$ & $\begin{array}{c}\text { Pilosocereus } \\
\text { catingicola }\end{array}$ & $\begin{array}{c}\text { Harrisia } \\
\text { adscendens }\end{array}$ & $\begin{array}{l}\text { Pilosocereus } \\
\text { gounellei }\end{array}$ & $\begin{array}{l}\text { Melocactus } \\
\text { bahiensis }\end{array}$ & $\begin{array}{c}\text { Opuntia } \\
\text { palmadora }\end{array}$ & \\
\hline Mischocyttarus lanei & 1 & 1 & 0 & 0 & 0 & 0 & 2 \\
\hline Polistes billardieri & 1 & 4 & 2 & 3 & 0 & 0 & 10 \\
\hline Polistes canadensis & 142 & 32 & 8 & 28 & 1 & 1 & 212 \\
\hline Polistes simillimus & 6 & 9 & 0 & 1 & 2 & 0 & 18 \\
\hline Polistes versicolor & 14 & 27 & 52 & 5 & 1 & 0 & 99 \\
\hline Polybia ignobilis & 33 & 35 & 17 & 46 & 2 & 0 & 133 \\
\hline Polybia occidentalis & 43 & 12 & 0 & 14 & 1 & 0 & 70 \\
\hline Polybia paulista & 21 & 29 & 30 & 26 & 0 & 0 & 106 \\
\hline Polybia sericea & 46 & 194 & 3 & 12 & 2 & 1 & 258 \\
\hline Total & 307 & 343 & 112 & 135 & 9 & 2 & 908 \\
\hline
\end{tabular}

Tabela II. Sobreposição de nichos tróficos entre espécies de vespas sociais que forragearam em frutos de cactos em Ipirá, Bahia, de setembro de 1999 a maio de 2000.

\begin{tabular}{|c|c|c|c|c|c|c|c|}
\hline & P. paulista & P. occidentalis & $P$. sericea & P. ignobilis & P. canadensis & P. versicolor & P. simillimus \\
\hline Polybia paulista & - & & & & & & \\
\hline Polybia occidentalis & 0,3714 & - & & & & & \\
\hline Polybia sericea & 0,2364 & 0,2326 & - & & & & \\
\hline Polybia ignobilis & 0,3910 & 0,2481 & 0,2632 & - & & & \\
\hline Polistes canadensis & 0,3208 & 0,2877 & 0,1557 & 0,3255 & - & & \\
\hline Polistes versicolor & 0,0446 & 0,2020 & 0,4141 & 0,2020 & 0,1919 & - & \\
\hline Polistes simillimus & 0,0556 & 0,3889 & 0,5000 & 0,0556 & 0,3889 & 0,0000 & - \\
\hline Polistes billardieri & 0,2778 & 0,0556 & 0,5000 & 0,3889 & 0,0556 & 0,2778 & 0,5000 \\
\hline
\end{tabular}

seleção dos recursos alimentares, apresentando alta equitabilidade na utilização de cada fonte de recurso $\left(J^{\prime}=0,99\right.$ e 0,86 respectivamente), enquanto $P$. sericea $\left(J^{\prime}=0,43\right), P$. canadensis $\left(\mathrm{J}^{\prime}=0,56\right)$ e $P$. versicolor $\left(\mathrm{J}^{\prime}=0,72\right)$ forragearam preferencialmente em uma única espécie de cacto (Tab. III).

Polistes canadensis foi a espécie com maior número de ninhos na área de estudo ( $\mathrm{n}=102$; densidade de 1,70 ninhos/ ha) e $P$. ignobilis a espécie com menor número de ninhos ( $\mathrm{n}=1$; densidade de 0,02 ninhos/ha) (Tab. IV). Espécies com colônias numerosas, como P. ignobilis, Polybia occidentalis (Olivier, 1791), $P$. paulista e $P$. sericea, apesar do pequeno número de ninhos encontrados (21 ninhos; $10,82 \%$ do total), representaram a maioria das vespas encontradas forrageando em Cactaceae (567 indivíduos; $62,44 \%$ do total). Esses dados corroboram os obtidos por HenRiques et al. (1992), que sugerem que a baixa densidade de ninhos de algumas espécies de vespas sociais no cerrado, ocorra em função da existência de colônias com grande número de indivíduos.

A densidade total de ninhos de vespas na Caatinga de Ipirá ( $\mathrm{D}=3,23$ ninhos/ha) foi menor que a encontrada por Henriques
Tabela III. Número de indivíduos (N), amplitude de nicho $\left(\mathrm{H}^{\prime}=\right.$ índice de Shannon-Winner) e equitabilidade (J' = Pielou) de vespas sociais forrageando em frutos de cactos em uma área de Caatinga em Ipirá, Bahia, de setembro de 1999 a maio de 2000.

\begin{tabular}{lrcc}
\hline \multicolumn{1}{c}{ Vespas } & $\mathrm{N}$ & $\mathrm{H}^{\prime}$ & $\mathrm{J}^{\prime}$ \\
\hline Mischocyttarus lanei & 2 & 0,69 & 1,00 \\
Polistes billardieri & 10 & 1,08 & 0,96 \\
Polistes canadensis & 212 & 1,01 & 0,56 \\
Polistes simillimus & 18 & 1,12 & 1,76 \\
Polistes versicolor & 99 & 1,17 & 0,72 \\
Polybia ignobilis & 133 & 1,38 & 0,86 \\
Polybia occidentalis & 70 & 0,98 & 0,71 \\
Polybia paulista & 106 & 1,38 & 0,99 \\
Polybia sericea & 258 & 0,78 & 0,43 \\
\hline
\end{tabular}

et al. (1992) em ambiente de Cerrado, no Brasil Central. No Cerrado, as densidades encontradas variaram de $\mathrm{D}=1,00$ a 1,60 ninhos/ha (Polistes satan Bequaert, 1940 e Polybia paulista), en- 
quanto na Caatinga as densidades variaram de $\mathrm{D}=0,02$ a 1,70 ninhos/ha (Polybia ignobilis e Polistes canadensis) (Tab. IV).

Diferente do encontrado por Henriques et al. (1992), as vespas em ambiente de Caatinga apresentam preferência na escolha de substrato de nidificação (Teste de médias de Kruskal-Wallis, $\mathrm{p}$ $<0,05)$. Do total de 194 ninhos encontrados, 185 ninhos $(95,37 \%)$ foram estabelecidos em árvores e/ou arbustos espinhosos. Três espécies de vespas ( $P$. canadensis, $P$. versicolor e $P$. simillimus) nidificaram exclusivamente em cactos colunares (C. jamacaru e P. catingicola). SAntos \& GobBi (1998), estudando os hábitos de nidificação de $P$. canadensis em uma área de Caatinga, encontraram preferência da espécie em nidificar em cactáceas. Para esses autores, essa preferência por nidificar em substratos espinhosos está relacionada com a proteção das colônias contra agentes predadores. SмITH (2004) e CRuz et al. (2006), trabalhando em florestas tropicais úmidas, também encontraram exemplos de vespas sociais que apresentam seleção de substrato de nidificação. Para DejeAn et al. (1998), a arquitetura dos ninhos e a seleção de locais de nidificação das vespas sociais foram selecionadas por dois fatores principais: condições climáticas e predadores.

Frutos de cactos constituem importante fonte de recurso alimentar para as vespas sociais da Caatinga de Ipirá, sendo utilizados por todas as espécies encontradas na região. A importância dos frutos de cactos como fonte de recurso alimentar para as vespas sociais se torna explícita, quando são comparados os dados encontrados neste estudo (908 indivíduos de nove espécies) com os dados de estudos de visitação floral. SANTos et al. (2006), avaliando a guilda de vespas sociais visitantes de flores na Caatinga em Itatim, encontraram apenas 534 indivíduos de 13 espécies de vespas sociais.

A predominância de vespas se alimentando em frutos de C. jamacaru e P. catingicola pode estar relacionada com a maior abundância destas espécies em relação a outras Cactaceae na área estudada. As vespas sociais, assim como outros organismos generalistas, costumam forragear predominantemente na fonte de recurso mais abundante, independente de preferência e/ou seletividade. A condição generalista das vespas sociais confere a estes insetos a propriedade de não depender de um recurso específico, fazendo com que esses insetos evitem aquelas fontes em que a disponibilidade de alimento não é constante (REAL 1981).

\section{AGRADECIMENTOS}

A Flávio França (UEFS) e à Daniela Zappi (Royal Botanic Gardens, Kew) pelo auxílio na identificação das espécies de cactos; a Carlos A.B. dos Santos Filho, Luiz C. de O. Pereira e Danovan de M. Novaes, pelo auxílio na coleta dos dados. A Carlos A.B. dos Santos, proprietário da Fazenda Encantado, pela permissão e facilidades oferecidas para a realização do estudo em sua propriedade.

\section{REFERÊNCIAS BIBLIOGRÁFICAS}

BEgon, M.; J.L. HAPER. \& C.R. TOWNSEND. 1996. Ecology. Individuals, populations and communities. Oxford, Blackwell, $3^{\text {rd }}$ ed., 912p.

CEI. 1994. Informações básicas dos municípios baianos: Região Paraguaçu, Salvador. Salvador, Centro de Estatística e Informações Bahia, 761p.

Cruz, J.D.; E. Giannotti; G.M.de M. Santos; C.C. Bichara Filho \& A.A. Rocha. 2006. Nest site selection and flying capacity of Neotropical wasp Angiopolybia pallens (Lepeletier, 1836) (Hymenoptera-Vespidae) in the Atlantic Rain Forest, Bahia State, Brazil. Sociobiology 47 (3): 739-750.

Dejean, A.; B. Corbara \& J.M. Carpenter. 1998. Nesting site selection by wasps in the Guianese rain forest. Insects Sociaux 45 (1): 33-41

Henriques, R.P.B.; I.R. Diniz \& K. Kitayama. 1992. Nest density of some social wasp species in Cerrado Vegetation of Central Brazil (Hymenoptera: Vespidae). Entomologia Generalis 17 (4): 265-268.

Hermes, M.G. \& A. Kohler. 2006. The flower-visiting wasps (Hymenoptera, Vespidae, Polistinae) in two areas of Rio Grande do Sul State, southern Brazil. Revista Brasileira de Entomologia 50: 268-274.

Tabela IV. Número e densidade de ninhos de vespas sociais na Caatinga de Ipirá, Bahia, de setembro de 1999 a maio de 2000.

\begin{tabular}{lccc}
\hline \multicolumn{1}{c}{ Vespas } & Total de ninhos observados & Densidade média de ninhos ativos/ha & Número estimado de indivíduos por colônia \\
\hline Mischocyttarus lanei & 2 & 0,03 & 2 \\
Polistes billardieri & 14 & 0,23 & 05 \\
Polistes canadensis & 102 & 1,70 & 11 \\
Polistes simillimus & 17 & 0,28 & 15 \\
Polistes versicolor & 38 & 0,63 & 16 \\
Polybia ignobilis & 1 & 0,02 & 4000 \\
Polybia occidentalis & 2 & 0,03 & 230 \\
Polybia paulista & 3 & 0,05 & 250 \\
Polybia sericea & 15 & 0,25 & 800 \\
\hline Total & 194 & 3,23 & \\
\hline
\end{tabular}


LIMA, J.L.S. DE. 1996. Plantas forrageiras das caatingas - usos e potencialidades. Petrolina, EMBRAPA-CPATSA, PNE, RBGKEW, 37p.

Machado, V.L.L. 1982. Plants which supply "hair" material for nest building of Protopolybia sedula (Saussure, 1854), p. 189192. In: P. JAISSON (Ed). Social insects in tropics. Paris, University Paris-Nord, 280p.

Marques, O.M. \& C.A.L. De Carvalho. 1993. Hábitos de nidificação de vespas sociais (Hymenoptera, Vespidae) no município de Cruz das Almas, Estado da Bahia. Insecta 2 (2): 23 40.

Pereira, V.S. \& G.M. De M. SAntos. 2006. Diversity in bee (Hymenoptera: Apoidea) and social wasp (Hymenoptera: Vespidae: Polistinae) community. Neotropical Entomology 35 (2): 165-174.

Prado, D.E. \& P.E. Gibbs. 1993. Patterns of species distributions in the dry seasonal Forest of South America, Annals of the Missouri Botanical Garden 80: 902-927.

REAL, L.A. 1981. Uncertainty and pollinator-plant interactions: the foraging behavior of bees and wasps on artificial flowers. Ecology 62 (1): 20-26.

Santos, G.M. DE M. \& N. Gobbi. 1998. Nesting habits and colo- nial productivity of Polistes canadensis canadensis (L.) (Hymenoptera, Vespidae) in a Caatinga area, Bahia State, Brazil. Journal of Advanced Zoology 19: 63-69.

Santos, G.M.de M.; S.O.C. Silva; C.C. Bichara Filho \& N. Gobbi. 1998. Influencia del tamaño del cuerpo en el forrajeo de avispas sociales (Hymenoptera, Polistinae) visitantes de Syagrus coronata (Martius) (Arecacea). Revista Gayana de Zoologia 62: 167-170.

Santos, G.M.de M.; C.M.L. Aguiar \& N. Gobbi. 2006. Characterization of the social wasp guild (Hymenoptera, Vespidae) visiting flowers in the Caatinga (Itatim, Bahia, Brazil). Sociobiology 47 (2): 1-12.

Schoener, T.W. 1982. The controversy over interspecific competition. American Naturalist 70: 586-595.

Shannon, C.E. 1948. The mathematical theory of communication, p. 3-91. In: C.E. Shannon \& W. Weaver (Eds). The mathematical theory of communication. Urbana, University Illinois Press, 117p.

SмIтH, E.F. 2004. Nest sites of the paper wasp Mischocyttarus collarellus (Hymenoptera: Vespidae: Polistinae) in a Lowland Tropical Rain Forest. Journal of the Kansas Entomological Society 77 (4): 457-469.

Recebido em 27.IV.2007; aceito em 20.XI.2007. 